\title{
VALVULAR ACTION IN THE EMBRYONIC CHICK HEART BY LOCALIZED APPOSITION OF ENDOCARDIAL MASSES
}

\author{
BRADLEY M. PATTEN, THEODORE C. KRAMER AND \\ ALEXANDER BARRY \\ Department of Anatomy, University of Michigan Medical School \\ SIX FIGURES
}

The heart of a chick embryo at the time the blood first starts to circulate through it is essentially a double-layered tube. Its endocardial layer consists of an endothelial lining held in place within the outer epimyocardial investment by a surprisingly thick layer of gelatinous, non-cellular material which Davis ('24, '27) called cardiac jelly. Blood enters this primitive young heart at its caudal end and is forced out its cephalic end by peristaltoid contractions of the myocardium. The fact that the cardiac tube soon becomes bent on itself does not at first essentially change the way the blood passes through its unchambered length. Nor does this early tubular heart have anything resembling valves as we know them in the adult heart. It is not surprising, therefore, that when the recently established circulation is studied in living embryos one sees a very jerky progression of the corpuseles in the vessels entering and leaving the heart. There is with each cardiac cycle what one might call a "backlash." This term in this connection is meant to convey the idea that toward the end of each contraction cycle the corpuscles in an artery lose a little of the progression they have made under the thrust of systole. In veins a similar blacklash occurs as contraction commences at the atrial end of the cardiac tube. 
Early in the third day of incubation the corpuscles begin to progress with less and less jerkiness. The change is so gradual that one hardly notices it in watching the same embryo through the transition period. If, however, an embryo after the change, is directly compared with one before the change has occurred, the contrast is quite striking. In the older embryo, between each beat, the corpuscles seem to coast to a momentary pause without any of the retrogression seen in younger embryos. Their whole progression appears smoother, and the nature of their movement suggests the operation somewhere in the system of a valvular mechanism guarding against back flow. Yet, as already emphasized, there is no structure in the embryonic heart at this age which even remotely resembles the usual flap-like type of valve seen in the adult or in older embryos. Careful restudy of films recording the cardiac activity at the critical stages of development first suggested the answer to the change in character of blood movement. At the level of transition from atrium to ventricle, "slow-motion" pictures showed the endocardium in the atrioventricular eanal heaping up in "mounds" so that at the close of each contraction cycle the mounds made contact with each other and completely blocked the cardiac lumen. During this period of closure careful study of both film records and living embryos revealed no back flow of blood from the contracting ventricle into the atrium.

The non-committal term endocardial mounds is used in describing this phenomenon rather than endocardial cushions because at the ages when the closure first becomes apparent the material which is heaped up is non-cellular cardiac jelly (plate 1, A). During the third day cells grow into the cardiac jelly and it gradually becomes converted into a plastic young. connective tissue of the type commonly described as endocardial cushion tissue. Contrary to the conclusions of Chang ('32, p. 225), the source of the cells involved appeared to us to be the endothelium. Careful scrutiny of the endothelial lining in embryos just before cellular invasion of the cardiac jelly is due to commence reveals occasional local doubling of the 
ordinarily single cell layer (see place indicated by arrow in plate 1, A). The picture suggests a recent cell division with one of the cells moving out of the endothelial layer toward the cardiac jelly. Slightly older embryos present conditions consonant with such an interpretation. Sprawling mesenchymal cells can be seen in the cardiac jelly adjacent to the endothelium, whereas none have as yet reached the cardiac jelly adjacent to the myocardium (plate $1, B$ ). A well-graded series of embryos is necessary if this intermediate stage is to be observed for once the cellular invasion has started it spreads rapidly all the way through the cardiac jelly to the myocardium (plate $1, \mathrm{C}$ ). One gets the impression from seeing a series of fixed preparations that the cardiac jelly serves as a supporting medium through which these cells move. It should be emphasized, however, that the lace work of strands along which the cells appear to be moving undoubtedly is a distorted representation incident to the coagulation of a substance which in the living embryo was gelatinous and more nearly homogeneous. Further studies are being undertaken in an effort to learn more of its true physical and chemical properties.

While this alteration in endocardial histology is occuring, study of living embryos shows relatively little change in the manner in which closure of the cardiac lumen is effected by the mounds. As endocardial cushion tissue replaces the cardiac jelly, the mounds become more sharply circumscribed and the closure is perhaps a little more positive, but the essential mechanism of blocking the cardiac lumen against regurgitation by the heaping up of a mound of plastic material remains unchanged.

This curious action of the endocardial mounds was noted in some of our earliest films which were made between 1928 and 1930. At about that time it was pointed out to Dr. Bremer on the occasion of a visit he made to us in Cleveland, where this work was begun. Dr. Bremer subsequently, in his own material, confirmed these early observations (personal communication) and mentioned them in connection with his work 
on circulatory disturbances in chick embryos (Bremer, '32b, p. 277). We know of nothing on the subject in the literature other than Bremer's brief comment and a surmise by Fischel ('29, p. 698) with reference to much older embryos to the effect that the endocardial cushions of the atrioventricular canal might be apposed to each other during systole and so exert a valvular action. Repeatedly, since it was first noticed, we have seen this same phenomenon both in living embryos and in micro-moving picture records of embryonic heart action. It has been mentioned in lectures given in connection with the showing of our films, but as far as we are aware, it is not a generally recognized phenomenon and has never been described in any detail. The writing up of these observations has been delayed pending the securing of additional film which would show more clearly certain phases of the changes involved. Some of our recently obtained records seemed to meet this need and led to the publication of this long postponed account.

Aside from seeing the living heart, or moving pictures of it in action, probably the easiest way of understanding the manner in which these endocardial mounds operate is afforded by superimposing tracings made by projecting through a photographic enlarging apparatus frames of a moving picture film showing different phases of the action. Such tracings showing the endocardial mounds in their open and closed position at the level of the atrioventricular canal in the heart of a chick of about 50 hours of incubation are reproduced in figure 1 . The embryo was mounted so it could be viewed from the left, an orientation which at this age gives the best exposure of the atrioventricular canal. The solid outline represents the external contour of the heart in diastole. The broken lines show the external configuration during systole. The positions of the endocardial mounds, separated as they appear during the filling of the ventricle, are indicated by the beaded lines. The positions of the endocardial mounds as they are apposed, blocking the atrioven- 
tricular canal against regurgitation of blood, are indicated by dotted lines.

This peculiar type of valvular action was noticed first at the atrioventricular canal. But as soon as the heart action was restudied in the light of this finding it became apparent that there was a second region where closure was effected by a similar mechanism. This was at the transition from ventricular conus to truncus arteriosus - in other words, at

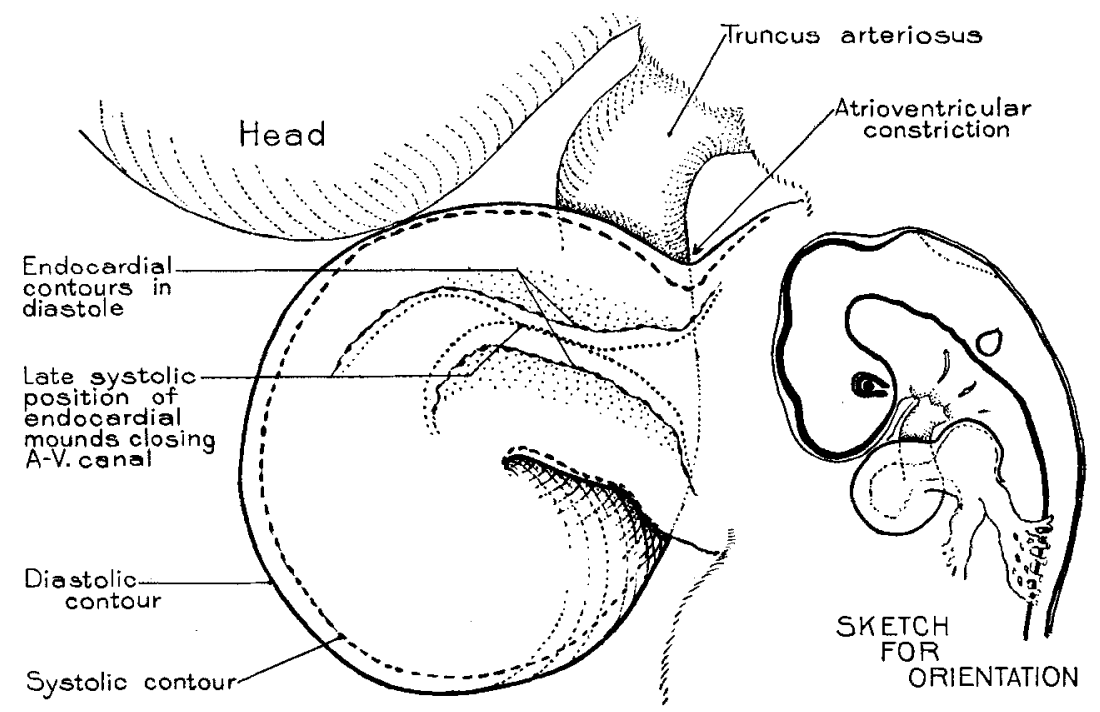

Fig. 1 Superimposed tracings made by projecting frames from moving picture film showing the heart action of a 50-hour chick. The solid external outlines and the beaded endothelial contours show conditions when blood is passing through the atrioventricular canal. The broken external outline and the dotted endothelial contours show conditions when the endocardial mounds are apposed to each other, blocking the lumen against back flow.

approximately the level where the semilunar valves of the aorta and the pulmonary trunk are destined to be formed. It is interesting that this curious primitive type of valvular action should be manifested at this particular level even before the truncus is partitioned into aortic and pulmonary outlets and, of course, long before the button-like endocardial cushion tissue primordia of the leaflets of the semilunar valves are 
as much as suggested morphologically. This situation at the ventricular outlet is, however, in line with what we have seen happening at the atrioventricular canal. There an effective closure occurs before the endocardial masses that later divide the canal into right and left channels have acquired the ingrowing cells which give them their characteristic histological structure. In this location, also, anything presaging the formation of the definitive flap-like atrioventricular valves lies far in the future, speaking developmentally.

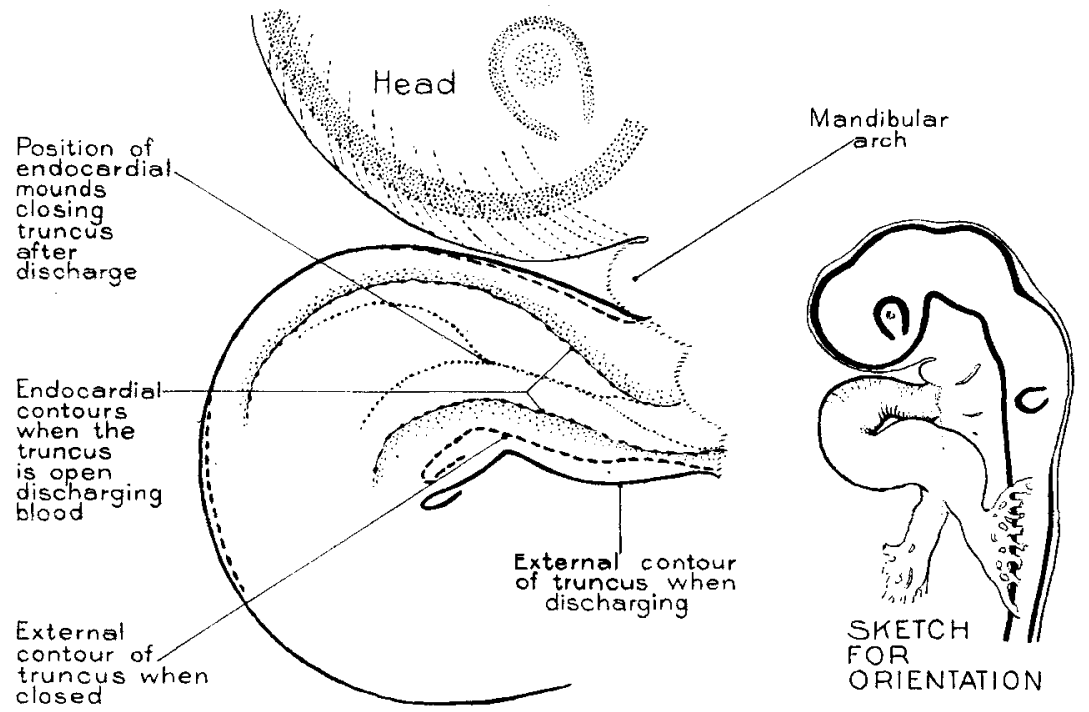

Fig. 2 Superimposed projection tracings showing closure by endothelial mounds at the transition from conus to truneus arteriosus. Scheme of representation similar to that employed in figure 1.

The closure at the base of the truncus arteriosus can readily be seen in a chick of about 48 to 50 hours. (Age scale as indicated in Patten, B. M., '22.) At this age there is considerable individual variability as to how tight the cardiac loop is twisted. An embryo in which the loop is rather loose, as indicated in the orienting diagram in figure 2, leaves the truncus projecting free of the body and in favorable position for observation and photography. The outlines of the truncus shown in figure 2 were made in the same manner as those of 
the atrioventricular canal shown in figure 1. Frames were selected for superimposition which showed the endocardial mounds separated from each other as they appear during systole when blood is being forced through the truncus, and as they appear when they close the truncus against back flow at the close of each ventricular systole.

The details of the action of the endocardial mounds of the truncus during one complete cardiac cycle are shown by the 12 tracings of figure 3 . These tracings were made from successive frames of film taken at the usual speed for silent moving pictures so that they represent the changes that have occurred at intervals of $1 / 16$ of a second. The dilation of the part of the ventricular lumen just proximal to the point of closure shown in the projections of frames 6 to 10 is interesting. It indicates the building up of pressure by a new contraction wave which has started at the sino-atrial end of the heart (Patten, B. M. and Kramer, T. C., 1933) and is "charging" the ventricle with blood. As this contraction wave advances the blood in the ventricle will force apart the truncus mounds (frames 11 and 12, and 1 to 3), and spurt out into the aortic roots. When the contraction wave reaches the truncus the lumen is closed, and the cardiac jelly tends for a brief time (frames 5 to 9) to be heaped up between the back pressure from the aortic system and the mounting pressure due to the recharging of the ventricle as a new cardiac cycle is initiated at the sino-atrial end of the heart. The effectiveness of these opposing pressures in heaping up the mounds is further indicated by the way the mounds flatten when the truncus is open, transmitting blood (frames 11 and 12 and 1 to 3 ). The rate of progression of the contraction wave at this stage of development is such that the atrioventricular mounds are closed as the ventricle is forcing blood out of the truncus, and the truncus mounds are closed as the atrioventricular mounds open to let a new charge of blood into the ventricle for the next cardiac cycle. The reciprocal action of the endocardial mounds at these two points 
in the cardiac tube thus provides a simple but surprisingly smoothly operating valvular mechanism.

Perhaps more interesting than the valvular action itself are some of the possible theoretical implications involved. It is well known that an excised chick heart kept alive by tissue eulture methods spends its energies in beating and, to

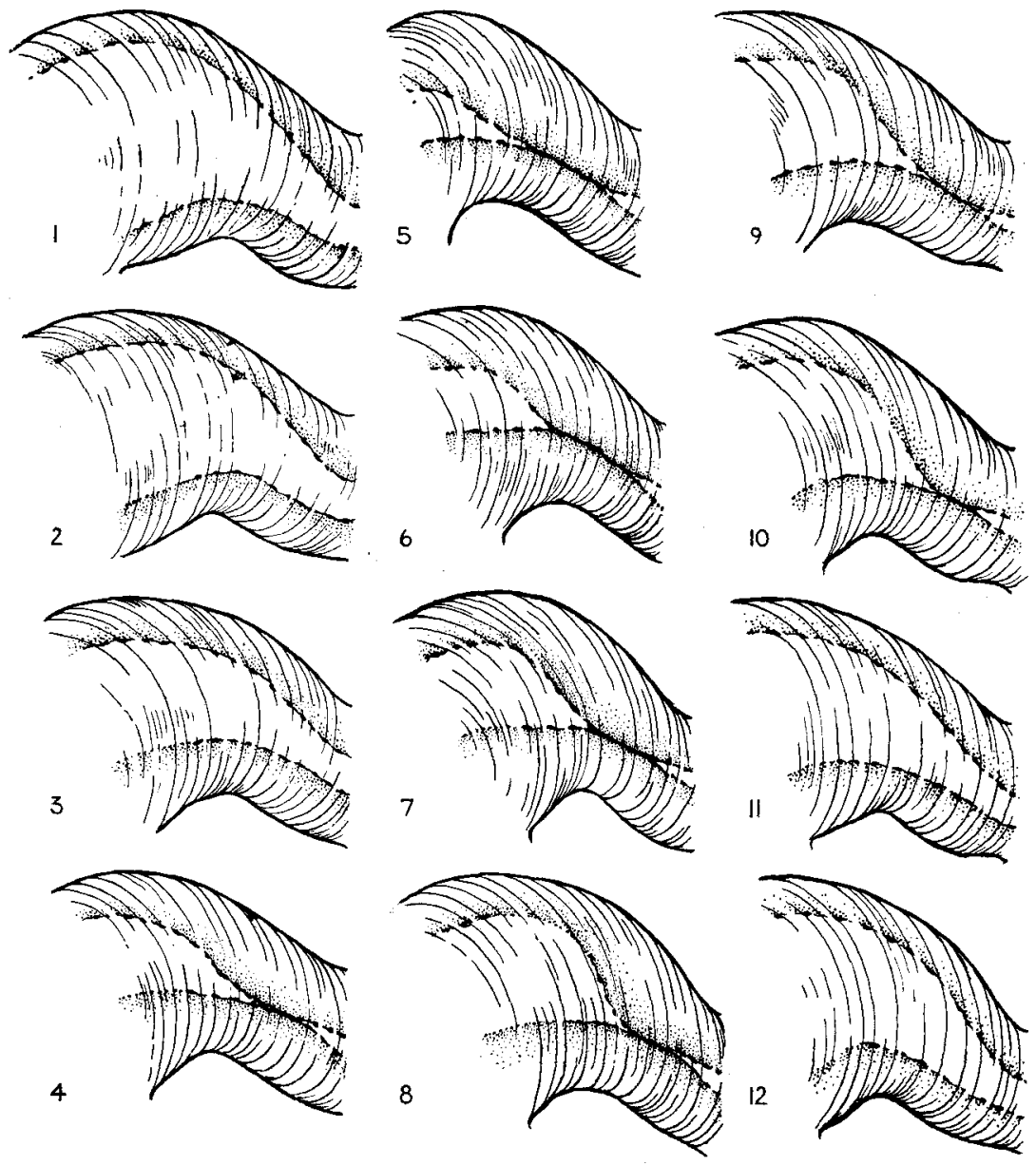

Fig. 3 Projection tracings of successive frames in a film analyzing closure at the cono-truncal region diagrammed in figure 2. The exposures were made at the rate of 16 per second and the 12 frames shown cover one complete cardiac cycle, from open phase to open phase. 
a less extent, in unorganized growth of its tissue elements. It does not, as do many other explanted parts of the growing body, differentiate morphologically under the influence of its "inherent developmental potencies." This would naturally lead one to turn inquiringly toward the possible moulding influences exerted by the hydrodynamic effects of blood currents and eddies. The most thoughtful approach to the moulding effects on the chick heart which might possibly be exterted by the blood pouring through it has been made by Bremer ('28, '32a). If, as Bremer contends, the blood passes through the bent tubular heart as spiral streams which maintain their identity sufficiently to have an internal moulding effect, the cardiac jelly may be far more important in the differentiation of the heart than anyone has suspected. Its consistency appears to be such that it would be far more readily moulded by blood currents than would any of the organized tissues. Should this prove to be the case, the form taken, and the distribution acquired, by the cardiac jelly could well set the pattern followed by endocardial cushion tissue when it takes over, by cellular invasion, the areas which were occupied by the cardiac jelly of younger embryos. Thus it is possible that the pattern of the endocardial ridges and mounds that are so important in the formation of the septa and valves of the developing heart might be dependent on the previous hydrodynamic moulding effect of blood currents on a non-cellular gelatinous material.

Furthermore, it seems probable that the same sort of material that we call cardiac jelly when we encounter it between the endothelium and the myocardial sleeve of the tubular heart is of general occurrence in the spaces between the primary germ layers of young embryos. In sections one can trace the same sort of coagulated meshwork from the endocardial spaces, out between the two layers of the dorsal mesocardium and into the space between the pharynx and the ectoderm. Furthermore, a watery body fluid if under appreciable pressure would stretch out the superificial ectoderm into formless rotundity, or if under low pressure would permit 
it to sag limply against underlying structures. A gelatinous material like cardiac jelly between ectoderm and entoderm would account for the way young embryos retain their characteristic shape when they are removed, and even when they are transected. It would also furnish a medium of such a consistency that mesenchymal cells might migrate in it instead of being limited in their excursions to amoeboid movement along a formed substratum such as the outer wall of the neural tube or the inner surface of the superficial ectoderm. The more one considers the places one finds mesenchymal cells in the growing embryo, the more inevitable it seems that there must be a material of just such properties through which they move. Such a conception is certainly an intriguing challenge to further experimental work.

\section{LITERATURE CITED}

BREMER, J. L. 1928 An interpretation of the devalopment of the heart. Am. J. Anat., 42: Part I, 307-337.

1932a The presence and influence of two spiral streams in the heart of the chick embryo. Am. J. Anat., 49: 409-440.

$1932 \mathrm{~b}$ Cireulatory disturbances in operated chick embryos: Reversal of heart beat. Anat. Ree, 51: 275-284.

ChANG, C. 1932 On the reaction of the endocardium to the blood stream in the embryonic heart, with special reference to the endocardial thickenings in the atrioventricular canal and the bulbus cordis. Anat. Rec., 5.1: $253-265$.

Davis, C. L. 1924 The cardiac jelly of the chick embryo. Anat. Rec., 27: 201202.

1927 Development of the human heart from its first appearance to the stage found in embryos of 20 paired somites. Carnegie Cont. to Emb., 19: 245-284.

Fischel, A. 1929 Lehrbuch der Entwicklung des Menschen. Julius Springer, Berlin, viii \& $822 \mathrm{pp}$.

Parten, B. M. 1922 The formation of the cardiac loop in the chick. Am. J. Anat., 30: 373-397.

Pat'Ten, B. M., and Kramer, T. C. 1933 The initiation of contraction in the embryonie chick heart. Am. J. Anat., 53: 349-375. 


\section{PLATE}


PLATE 1

\section{EXPLANATION OF FIGURES}

Drawings showing stages in the cellular invasion of cardiac jelly with the resultant formation of endocardial cushion tissue. All the drawings were made from comparable areas in the cono-truneus to the same scale ( $\times 1000$ original, reproduced $\times 750$ ).

A. From a 14-somite ( \pm 36 hours) ehick showing the primary non-eellular character of the cardiac jelly. Note the thinness of endothelium except for the place indicated by the arrow.

B. From a 33-somite ( \pm 65 hours) ehick showing the beginning of cellular invasion. Note the thickened character of the endothelium and the fact that cells have not as yet reached the myocardium.

C. From a $40-41$-somite chick ( \pm 84 hours) showing cells throughout the territory formerly occupied by the cardiac jelly alone. 
B. M. PATTEN, T, C. KRAMER AND A. BARRY
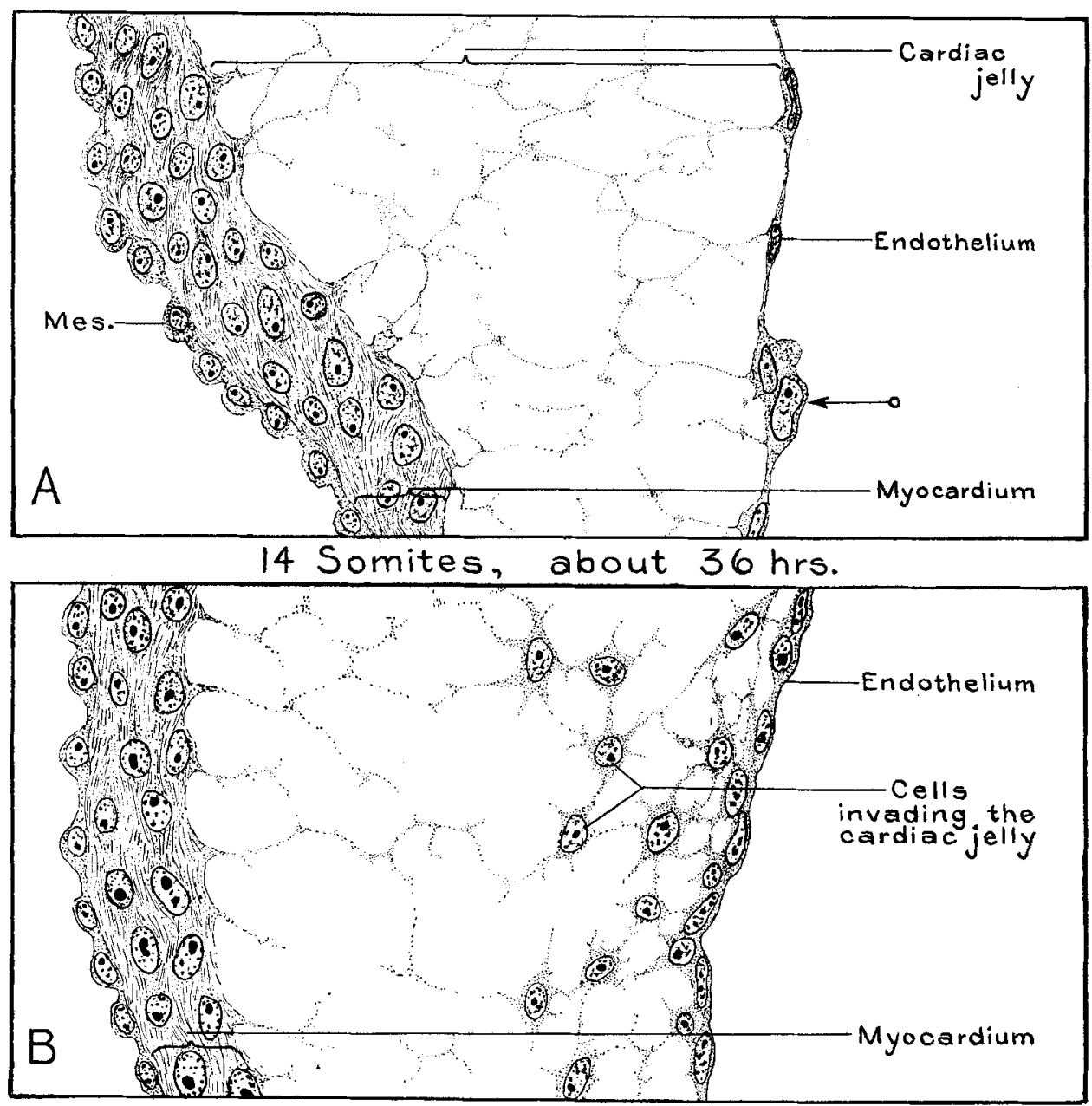

33 Somites, about $65 \mathrm{hrs}$.

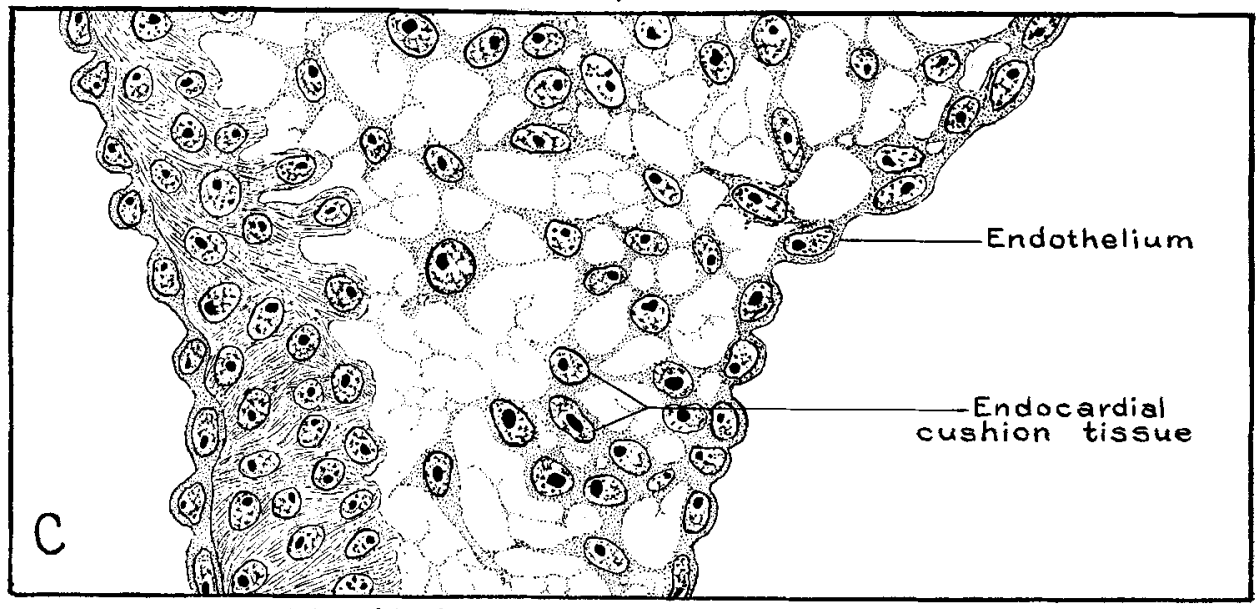

40-41 Somites, about $84 \mathrm{hrs}$. 